\title{
Effect of resistance exercise training on biochemical markers and anthropometric characteristics involved in atherosclerosis in obese women
}

\author{
Hossein TaheriChadorneshin ${ }^{1 *}$, Shila Neyebi-Far ${ }^{2}$
}

1. Department of Sport Sciences, University of Bojnord, Bojnord, Iran

2. Department of Sport Sciences, University of Sistan and Baluchestan, Zahedan, Iran

*Corresponding author:Tel: +98 5832284610 Fax: +98 5832284610

Address: Department of Sports Sciences, University of Bojnord, $4 \mathrm{Km}$ of Bojnord-Esfarāyen Highway, Bojnord, North Khorasan, Iran

E-mail: h.taheri@ub.ac.ir

Received; 2017/01/19 revised; 2017/04/30 accepted; 2017/05/26

\begin{abstract}
Introduction: Obese individuals have elevated levels of inflammatory and cell adhesion molecules that can critically induce the occurrence of atherosclerosis. Aerobic exercise training reduces biochemical markers and anthropometric characteristics involved in atherosclerosis. However, little is known about the effect of resistance exercise training on these biomarkers. This study aimed to investigate the effect of eight weeks of resistance training on atherosclerosis biochemical markers and anthropometric characteristics in obese women.
\end{abstract}

Materials and methods: Fourteen obese women completed an 8-week resistance exercise training protocol with moderate intensity at 9 stations (exercise involving the major muscle groups: 3 sets of 8-15 repetitions of chest press, leg press, seated pulley rows, overhead press, seated leg press, leg curl, triceps extensions, biceps curls, and calf raises). Fasting blood samples were taken before and after the 8-week exercise training. Intercellular Adhesion Molecule1 (ICAM-1) and C-reactive protein (CRP) levels were measured using commercial kits by ELISA method. The data were analyzed using dependent t-test.

Results: Resistance training significantly reduced the levels of atherosclerosis biochemical markers, ICAM-1 and CRP ( $\mathrm{P}=0.001)$. Also, body fat percentage $(\mathrm{P}=0.001)$, waist: hip ratio, body mass and body mass index significantly decreased following resistance training $(\mathrm{P}=0.001)$. However, no significant change occurred in platelet counts $(\mathrm{P}=0.922)$.

Conclusion: Resistance training results in reduction of inflammatory biomarkers involved in atherosclerosis as well as body fat.

Keywords: Resistance exercise training, Intercellular adhesion molecule 1, Obese

\section{Introduction}

Although increased low-density lipoprotein (LDL-c) and decreased highdensity lipoprotein (HDL-c) are considered to determine the risks of cardiovascular disease, reports show people who suffer from cardiovascular disease despite normal levels of LDL-c and HDL-C (1-3). Meanwhile, a lot of research suggests that cardiovascular disease has inflammatory background and that systemic inflammation plays a pivotal role in the development of atherosclerosis (4-6). Accordingly, in the past decade, more attention has been focused on inflammatory markers as independent factors that predict incidence of cardiovascular disease.

Intercellular adhesion molecule-1 (ICAM1) and C-reactive protein (CRP) act as important inflammatory markers, which are associated with the pathogenesis of atherosclerosis $(4,7-8)$. 
Cell adhesion molecules exists in membrane-bound and soluble forms. Membrane intercellular adhesion molecule-1 (mICAM-1) with $90 \mathrm{kDa}$ expresses constitutively on endothelial and non-endothelial cells and is up-regulated by adrenergic stimulation $(4,10,11)$ oxidative products $(8,9)$, shear stress $(10$ 12 ), and inflammatory cytokines such as CRP $(4,11,12)$. Circulating soluble ICAM1 (sICAM-1) is thought to originate from proteolytic of membrane-bound ICAM-1 and has a lower molecular weight than mICAM-1 (13). mICAM-1 mediates the adherence and subsequent infiltration of circulating leukocytes across the vascular endothelium $(11,13)$. sICAM-1 represents its expression on endothelial cells (13) and therefore provides an easy-to-measure clinical marker of vascular inflammation and endothelial activation (11). Holding 224 amino acids, CRP is synthesized and released by hepatocytes in response to inflammation $(4,14)$. Increased levels of CRP following inflammatory conditions result in expression of ICAM-1 on endothelial cells (1). Similarly, increased production of free radicals following adherence of immune cells to ICAM-1 results in proliferation of smooth muscle cells and re-expression of ICAM-1 on endothelial cells $(4,8,15)$. This results in greater adherence of plaque and LDL particles to endothelial cells that finally leads to the development of a mature atherosclerotic plaque $(4,15,16)$.

Researchers have used different nutritional and exercise training approaches to reduce the activation of endothelial cells and subsequently inhibit the expression of adhesion molecules $(6-9,17-19)$. In this regard, vitamin E supplementation has been suggested to decrease monocyte chemotactic activity (20) and surface expression of ICAM-1 induced by high-fat diet as it reduces the inflammatory marker (16). Also, it has been shown that daily treadmill walking (70 to $85 \%$ of maximum heart rate) (6) and cycle ergometer training ( $80 \%$ of maximum heart rate) (7) with modified nutritional diet (high fiber, low fat) reduce ICAM-1 and CRP levels in diabetes mellitus type 2 and coronary artery disease, respectively $(6,7)$. Furthermore, both short- $(6,15)$ and longterm (21) aerobic exercise training with moderate intensity alters circulating ICAM-1 and CRP in sedentary obese and overweight adults with metabolic syndrome. Although 12-week home-based bicycle exercise training reduces serum ICAM-1 in patients with chronic heart failure, serum ICAM-1 returns to baseline upon detraining (8).

Aerobic exercise training reduces inflammatory markers involved in atherosclerosis in obese and overweight individuals in both pathological (16) and physiological conditions (14, 21). Except for one study (14), the effect of resistance exercise training (exercises that increase energy expenditure, basal metabolic rate, and body's muscle mass) on atherosclerosis biochemical markers has not been well studied. The annual rate of overweight and obesity have recently grown and can be seen in both sexes and all races (4). In a national study of diseases in Iran, cardiovascular disease was ranked third, and atherosclerosis had a higher prevalence in obese and overweight subjects (22). Furthermore, obese individuals have elevated levels of ICAM1 than lean individuals (23-26). The present study investigated the effects of 8 weeks of resistance training on the risk factors involved in atherosclerosis in obese women.

\section{Materials and methods}

Participants: The present quasiexperimental study was approved by the Human Subjects Protection Committee of the University of Birjand (Iran). Twenty sedentary and obese women with regular menstrual periods were chosen after they completed health and Physical Activity Readiness Questionnaire (PAR-Q). Also, physical fitness level was evaluated by Baecke's physical activity questionnaire. 
Given the proposition by Bielinski and colleague (2008) that ICAM-1 concentration in serum/plasma is heritable, our exclusion criteria included subjects with any infection disease, cardiovascular disease, atherosclerosis, hyperlipidemia, hypertension, diabetes, cancer, and family background of these diseases (13). Also, we excluded any subjects who smoked or used cholesterol-lowering medications and non-steroidal anti-inflammatory drugs. All of them signed a written informed consent knowing the potential benefits and associated risks. Six subjects were excluded due to lack of regular participation. Maximal oxygen consumption $\left(\mathrm{VO}_{2} \max \right)$ of subjects was determined by Storer maximal bicycle test before the exercise training protocol. Physical characteristics of the subjects are listed in Table 1.

Table 1. Subject characteristics.

\begin{tabular}{ll}
\hline Age $($ year $)$ & $36 \pm 6$ \\
Body mass $(\mathrm{kg})$ & $77 \pm 10$ \\
Hight $(\mathrm{cm})$ & $159 \pm 7$ \\
$\mathrm{BMI}\left(\mathrm{kg} / \mathrm{m}^{2}\right)$ & $30.47 \pm 4.28$ \\
$\mathrm{VO}_{2} \mathrm{max}, \mathrm{ml} \cdot \mathrm{kg}^{-1} \cdot \mathrm{min}^{-1}$ & $18.23 \pm 3$ \\
$\mathrm{BF} \%$ & $35.93 \pm 3.36$
\end{tabular}

Values are presented in means \pm standard deviation. BMI, Body mass index; $\mathrm{VO}_{2} \max$, maximal oxygen consumption; $\mathrm{BF} \%$, body fat percentage.

Anthropometric measures: Subjects' height and body mass were measured by digital Stadiometer Seca. Body mass index (BMI) was calculated as weight in kilograms divided by the square of height in meters. Waist-hip ratio (WHR) was calculated as waist measurement (at the smallest circumference of the waist, just above the belly button) divided by hip measurement (the widest part of the buttocks). Skinfold thickness was obtained with skinfold caliper (Yagami model, Japan) on the right side of the subject's body. A 3-site skin fold equation of Jackson and Pollock (triceps, thigh, and suprailiac) was used to estimate body density, and body fat percentage (BF \%) was subsequently calculated using the Siri equation.

Determination of one-repetition maximum: One-repetition maximum (1RM) was determined before, at the fourth week of training, and 3 days after the last resistance exercise training (27). All strength tests throughout the study were conducted using the same equipment with identical positioning of the participant and controlled by the same investigator (28). Strength tests were always preceded by $7 \mathrm{~min}$ warm-up on a cycle ergometer. After warm-up, the subjects rested for 3 min. Weight was chosen according to the participant so that the participants lifted the weights at least once and up to 10 times. 1RM of agonist and antagonist muscles were measured on two separate days (27). Finally, 1RM was determined by Brzycki equation as follows: $1 \mathrm{RM}=$ Weight $\div[1.0278-(0.0278 \times$ Number of repetitions)]

Resistance exercise training: Participants were appropriately trained under physician supervision. They performed resistance exercise training with moderate intensity for 8 weeks ( 1 session per day, 4 days per week, at 50 to $65 \%$ of 1-RM). Resistance exercise training was conducted at 9 stations (exercise involving the major muscle groups: chest press, leg press, seated pulley rows, overhead press, seated leg press, leg curl, triceps extensions, biceps curls, and calf raises). Each exercise was performed for 3 sets with 8 to 11 repetitions in the first 4 weeks and increased to 15 repetitions during the last 4 weeks. Exercise in each station lasted for 30 seconds and the rest between two stations was 120 seconds. One exercise session consisted of 15 to 20 min warmup, $30 \mathrm{~min}$ resistance exercise training, and 10 min cool-down.

Blood sampling and biochemistry assay: Twelve-hour fasting blood samples were obtained from the antecubital vein before and 48 hours after the last resistance exercise training (14). To avoid facing the hormonal changes of the menstrual cycle, 
sampling time coincided with the luteal phase because estrogen acts as a reducing agent of serum ICAM-1 (26). The samples were centrifuged (Eppendorf Centrifuge, Mini SpinR, Germany) for $10 \mathrm{~min}$ at 3000 $\times g$ at $4{ }^{\circ} \mathrm{C}$. Serums were collected and stored immediately at $-80{ }^{\circ} \mathrm{C}$. ICAM-1 (Gen-Probe Diaclone SAS, France), and CRP (Mississauga, Ontario, Canada) levels in the serums were measured using the commercial ELISA kit according to the manufacturer's instructions. The sensitivity of the ICAM-1 and CRP kits were less than $8.68 \mathrm{ng} / \mathrm{ml}$ and $10 \mathrm{ng} / \mathrm{ml}$, respectively.

\section{Statistical analysis}

Statistical analyses were performed with Statistical Package for the Social Sciences (SPSS) version 16. Normality of data was confirmed by Shapiro-Wilk test. Data were analyzed using the paired t-test. All data are expressed as means \pm standard deviation. $\mathrm{P}$ values $<0.05$ were considered statistically significant.

\section{Results}

Mean and standard deviation of the dependent variables in the pre- and postresistance exercise training are displayed in Table 2. In the context of atherosclerosis biochemical markers, resistance exercise training significantly reduced serum levels of ICAM-1 in postcompared to pre-resistance exercise training $(\mathrm{P}=0.001)$. The serum levels of $\mathrm{CRP}$ reduced significantly following resistance exercise training $(\mathrm{P}=0.001)$. In terms of anthropometric characteristics, resistance exercise training significantly reduced body mass $(\mathrm{P}=0.001)$, $\mathrm{BMI}$ $(\mathrm{P}=0.001), \mathrm{BF} \% \quad(\mathrm{P}=0.001)$ and $\mathrm{WHR}$ $(\mathrm{P}=0.010)$. In contrast, resistance exercise training did not have a significantly effect on platelet counts $(\mathrm{P}=0.922)$.

Table 2. Values of dependent variables in pre- and post-resistance exercise training.

\begin{tabular}{lcccc}
\hline Dependent variables & \multicolumn{2}{c}{ Time } & t value & P value \\
\cline { 2 - 3 } & Pre & Post & \\
\hline Serum ICAM1 (ng/ml) & $661.90 \pm 163.19$ & $512.42 \pm 130.92$ & $10.5^{*}$ & 0.001 \\
Serum CRP (ng/ml) & $2871.64 \pm 2413$ & $2443.91 \pm 2023.14$ & $9.74 *$ & 0.001 \\
Platelet counts (count/mm $\left.\mathrm{mm}^{3}\right)$ & $230360 \pm 65505$ & $229000 \pm 38454$ & 0.099 & 0.922 \\
Body mass $(\mathrm{kg})$ & $76.68 \pm 10.45$ & $73.92 \pm 10.26$ & $7.89 *$ & 0.001 \\
BMI (kg/m2) & $30.47 \pm 4.28$ & $29.40 \pm 4.16$ & $7.23^{*}$ & 0.001 \\
BF $(\%)$ & $35.93 \pm 3.36$ & $34.41 \pm 3.58$ & $5.08^{*}$ & 0.001 \\
WHR & $0.78 \pm 0.03$ & $0.77 \pm 0.03$ & $3.01 *$ & 0.010 \\
\hline
\end{tabular}

Values are presented in means \pm standard deviation. The asterisk $\left(^{*}\right)$ indicates a significant difference. ICAM-1, intercellular adhesion molecule-1; CRP, C-reactive protein; BMI, Body mass index; BF \%, body fat percentage; WHR, waist: hip ratio.

\section{Discussion}

In recent years, there has been a growing number of prospective epidemiologic studies to demonstrate that ICAM-1 and CRP are independent predictors of myocardial infarction, stroke, atherosclerosis, and sudden cardiac death, even in apparently healthy individuals $(4,5,8)$. In the present study, we showed that reduced levels of ICAM- 1 and CRP in obese women correspond with decreased levels of body fat.
There is now a substantial body of evidence suggesting that aerobic exercise training is a significant factor against incidence of atherosclerosis through reducing inflammatory markers (6-9). Here, we found reductions in ICAM-1 and CRP following resistance exercise training. The results of this study are consistent with studies that report a reduction in ICAM-1 and CRP levels, weight and BMI following both \pm exercise training and dietary modification $(6,7)$. By 
reducing fat intake, dietary modification is believed to affect adipose tissue that subsequently reduces ICAM-1 expression (21). Especially, decreased visceral adipose tissue in obese women results in greater reduction in inflammatory cytokines and ICAM-1 expression (21). In this context, a positive correlation has been showed between ICAMI, BMI and BF (\%) in type 2 diabetic patients (5). Also, this finding is consistent with the study of Puglisi and colleague (2008) which attributed a reduction in plasma ICAM-1 following 6-week walking to reduced inflammatory cytokines in overweight and postmenopausal women (9). As we found a decline in ICAM-1 in obese women, a reduction in ICAM-1 is reported in obese men after daily treadmill walking (6). Serum ICAM-1 level is not different between men and women (23). In contrast, 8 to 14 weeks of rowing on a home rowing ergometer (30) and stationary cycle training (12) did not have any significant effect on concentration of ICAM-1 and CRP. This lack of change can be attributable to the inability of the mentioned exercise training in reducing body fat. Although it seems that artery remodeling induced by exercise training results in less shear stress and ICAM-1 expression (15), it seems that the major determinant of changes in ICAM-1 and CRP levels is body fat, particularly visceral fat. By producing inflammatory cytokines of tumor necrosis factor (TNF- $\alpha$ ) and interleukin-1 (IL-1), adipose tissue increases expression of ICAM-1 on endothelial cells $(6,7,15,21)$. Inflammatory cytokines enhance activation of NF-kappaB that regulates transcription of ICAM-1 (4). Moreover, NF-kappaB promotes the attachment of monocytes and macrophages to vessel walls by synthesizing and releasing proinflammatory cytokines (4). Importantly, TNF- $\alpha$ and IL- 1 stimulate synthesis of CRP in hepatocyte, while CRP increases expression of ICAM-1 $(8,23)$.
By increasing anti-inflammatory cytokine, exercise training has been showed to $(9,14)$ reduce the expression of ICAM-1 and CRP induced by inflammatory cytokines of IL- 1 and TNF- $\alpha$ that release from adipose tissue. Furthermore, exercise training improves antioxidant capacity, subsequently reducing free radical production and LDL oxidation. Decreased expression of ICAM-1 is also associated with reduced oxidation of LDL (9). Finally, it seems that part of the observed decline in levels of ICAM-1 in the present study is due to reduced shear stress induced by angiogenesis and atherogenesis after exercise training $(12,15)$. Shear stress results in cleavage of mICAM-1 from endothelial cells and its release into blood circulation (12).

In contrast, resistance exercise training has no effect on the number of platelets. Immune cells transfer to adjacent tissue by binding to adhesion molecules, subsequently resulting in to increased free radicals' production and LDL oxidation. Oxidation of LDL ultimately increases expression of ICAM-1 in endothelial cells. Platelets stick to ICAM-1, whereby vascular narrowing and occlusion may occur (4). In one study, reduced expression of atherosclerosis biochemical markers was attributed to decreased adhesion of monocytes to cultured endothelial cells in overweight and obese subjects who conducted both exercise training and dietary modification (6). Effect of exercise training on endothelial function is mediated by increased levels of HDL. In turn, HDL stimulates prostaglandin from vessel wall or smooth muscle cells and thereby inhibits platelet aggregation, leading finally to reduce expression of ICAM-1 (31).

Due to study limitations, the activity of immune cells and platelet adhesion was not measured in the present study and is recommended to be considered in future researches. 


\section{Conclusion}

Resistance training results in reduction of inflammatory biomarkers involved in atherosclerosis by decreasing body fat.

\section{References}

1. Blake GJ, Ridker PM. Novel clinical markers of vascular wall inflammation. Circul Res. 2001; 89(9):763-71.

2. Siasos G, Tsigkou V, Oikonomou E, Zaromitidou $\mathrm{M}$, Tsalamandris $\mathrm{S}$, Mourouzis K, et al. Circulating biomarkers determining inflammation in atherosclerosis progression. Curr Med Chem. 2015; 22(22):2619-35.

3. Palmefors H, DuttaRoy S, Rundqvist B, Börjesson M. The effect of physical activity or exercise on key biomarkers in atherosclerosis-a systematic review. Atherosclerosis. 2014; 235(1):150-61.

4. Kon Koh K, Hwan Han S, Quon MJ. Inflammatory markers and the metabolic syndrome: insights from therapeutic interventions. J Am Coll Cardiol. 2005; 46(11):1978-85.

5. Tönjes A, Scholz M, Fasshauer M, Kratzsch J, Rassoul F, Stumvoll M, et al. Beneficial effects of a 4-week exerciseprogram on plasma concentrations of adhesion molecules. Diabetes Care. 2007; 30(3):e1.

6. Roberts CK, Won D, Pruthi S, Kurtovic S, Sindhu RK, Vaziri ND, et al. Effect of a short-term diet and exercise intervention on oxidative stress, inflammation, MMP-9, and monocyte chemotactic activity in men with metabolic syndrome factors. J Appl Physiol. 2006; 100(5):1657-65.

7. Sixt S, Beer S, Blu"her M, Korff N, Peschela T, Sonnabend M, et al. Longbut not short-term multifactorial intervention with focus on exercise training improves coronary endothelial dysfunction in diabetes mellitus type 2 and coronary artery disease. Eur Heart J. 2010; 31(1):112-9.

\section{Acknowledgments}

We thank the participants for their valuable assistance with us in carrying out the study.

8. Adamopoulos S, Parissis J, Kroupis C, Georgiadis M, Karatzas D, Karavolias $\mathrm{G}$, et al. Physical training reduces peripheral markers of inflammation in patients with chronic heart failure. Eur Heart J. 2001; 22(9):791-7.

9. Puglisi MJ, Vaishnav U, Shrestha S, Torres-Gonzalez M, Wood RJ, Volek JS, et al. Raisins and additional walking have distinct effects on plasma lipids and inflammatory cytokines. Lipids Health Dis. 2008; 14:1-9.

10. Rehman J, Mills PJ, Carter SM, Chou J, Thomas J, Maisel AS. Dynamic exercise leads to an increase in circulating ICAM-1: further evidence for adrenergic modulation of cell adhesion. Brain Behav Immun. 1997; 11(4):343-51.

11. von Känel R1, Preckel D, Kudielka BM, Fischer JE. Responsiveness and habituation of soluble ICAM-1 to acute psychosocial stress in men: determinants and effect of stresshemoconcentration. Physiol Res. 2007; 56(5):627-39.

12. Sabatier MJ, Schwark RH, Lewis R, Sloan G, Cannon J, Kevin McCully K. Femoral artery remodeling after aerobic exercise training without weight loss in women. Dyn Med. 2008; 13(7):1-8.

13. Bielinski SJ, Pankow JS, Foster CL, Miller MB, Hopkins PN, Eckfeldt JH, et al. Circulating soluble ICAM-1 levels shows linkage to ICAM gene cluster region on chromosome 19: The NHLBI family heart study follow-up examination. Atherosclerosis. 2008; 199(1):172-8.

14. Olson TP, Dengel DR, Leon AS, Schmitz KH. Changes in inflammatory 
biomarkers following one-year of moderate resistance training in overweight women. Int J Obes (Lond). 2007; 31(6):996-1003.

15. Rector RS, Turk JR, Sun GY, Guilford B, Toedebusch BW, Mcclanahon MW, et al. Short-term lifestyle modification alters circulating biomarkers of endothelial health in sedentary overweight adults. Appl Physiol Nutr Metab. 2006; 31(5):512-7.

16. Nappo F, Esposito K, Cioffi M, Giugliano G, Molinari AM, Paolisso $\mathrm{G}$, et al. Postprandial endothelial activation in healthy subjects and in type 2 diabetic patients: role of fat and carbohydrate meals. J Am Coll Cardiol. 2002; 39(7):1145-50.

17. Jalaly L, Sharifi G, Faramarzi M, Nematollahi A, Rafieian-Kopaei M, Amiri M, Moattar F. Comparison of the effects of Crataegus oxyacantha extract, aerobic exercise and their combination on the serum levels of ICAM-1 and E-Selectin in patients with stable angina pectoris. Daru. 2015; 23(1):54-61.

18. Steckhan N, Hohmann CD, Kessler C, Dobos G, Michalsen A, Cramer H. Effects of different dietary approaches on inflammatory markers in patients with metabolic syndrome: A systematic review and meta-analysis. Nutrition. 2016; 32(3):338-48.

19. Nayebifar S, Afzalpour ME, Kazemi T, Eivary SH, Mogharnasi M. The effect of a 10-week high-intensity interval training and ginger consumption on inflammatory indices contributing to atherosclerosis in overweight women. $\mathrm{J}$ Res Med Sci. 2016; 21:116-23.

20. Theriault A, Chao JT, Gapor A. Tocotrienol is the most effective vitamin $\mathrm{E}$ for reducing endothelial expression of adhesion molecules and adhesion to monocytes. Atherosclerosis. 2002; 160(1):21-30.

21. Ziccardi P, Nappo F, Giugliano G, Esposito K, Marfella R, Cioffi M, et al. Reduction of inflammatory cytokine concentrations and improvement of endothelial functions in obese women after weight loss over one year. Circulation. 2002; 105(7):804-9.

22. Naghavi M, Abolhassani F, Pourmalek F, Lakeh MM, Jafari N, Eshrati B. The burden of disease and injury in iran 2003. Popul Health Metr. 2009; 7(1):9.

23. Pontiroli AE, Pizzocri P, Koprivec D, Vedani P, Marchi M, Arcelloni C, et al. Body weight and glucose metabolism have a different effect on circulating levels of ICAM-1, Eselectin, and endothelin-1 in humans. Eur J Endocrinol. 2004; 150(2):195200.

24. You T, Arsenis NC, Disanzo BL, LaMonte MJ. Effects of exercise training on chronic inflammation in obesity. Sports Med. 2013; 43(4):24356.

25. Figueiredo L, Nunes RB, Marmett B, de Sá LB, Arbex AK. Antiinflammatory effects of physical exercise on obesity. Open $\mathbf{J}$ Endocr Metab Dis. 2017; 7(01):44-51.

26. El-Kader SM, Al-Dahr MH. Impact of weight loss on oxidative stress and inflammatory cytokines in obese type 2 diabetic patients. Afr Health Sci. 2016;16(3):725-733.

27. Bemben DA, Fetters NL, Bemben MG, Nabavi N, Koh ET. Musculoskeletal responses to high- and low-intensity resistance training in early postmenopausal women. Med Sci Sports Exerc. 2000; 32(11):1949-57.

28. Sardroodian M, Madeleine P, Voigt M, Hansen, EA. Frequency and pattern of voluntary pedalling is influenced after one week of heavy strength training. Hum Mov Sci. 2014; 36:58-69.

29. Timmons BW, Hamadeh BW, Tarnopolsky MA. No effect of shortterm $17 \beta$-estradiol supplementation in healthy men on systemic inflammatory responses to exercise. Am J Physiol. 2006; 291(2):285-90.

30. Scheede-Bergdahl C, Olsen DB, Reving D, Boushel R, Dela F. 
Cardiovascular disease markers in type 2 diabetes: the effects of a moderate homebased exercise training programme. Diab Vasc Dis Res. 2009; 6(4):291-6.
31. Lerch PG, Spycher MO, Doran JE. Reconstituted high densityi lipoprotein (rHDL) modulates platelet activity in vitro and ex vivo. Thromb Haemost. 1998; 80(2):316-20. 